\title{
ECTOPIC PREGNANCY : A MAJOR CAUSE OF MATERNAL MORTALITY?
}

\author{
Jha R*, Singh $M^{*}$, Rana A, Singh A*, Bastola S*
}

\section{ABSTRACT}

Seventyeight cases of ectopic pregnancy were studied retrospectively at Tribhuvan University Teaching Hospital during April 1993 - December 1996. The overall incidence of etopic pregnancy was $0.86 \%$ live births. Grandmultipara were found to be at a low risk for ectopic pregnancy. A history of infertility and previous pelvic surgery were positive risk factors. The most common presenting symptoms were abdominal pain $(90 \%)$, history of amenorrhoea $(46 \%)$, abnormal uterine bleeding $(40 \%)$. On examination adenexal tenderness (72\%), abdominal tenderness (68\%), pallor (53\%) and cervical excitation (33\%) were the most frequent findings. Adenexal mass was noted in $22 \%$ cases and in $35 \%$ cases the size of the uterus could not be assessed. Ultrasonography and transvaginal sonography proved to be valuable diagnostic aids. Two maternal deaths $(2.6 \%)$ due to ectopic pregnancy occurred in the hospital during this period.

Key Words: Ectopic pregnancy, risk factors, sonography.

\section{INTRODUCTION}

Ectopic pregnancy is one of the leading causes of maternal morbidity and mortality. The risk of death from an extrauterine pregnancy is 10 times greater than from a vaginal delivery and 50 times greater than for an induced abortion. ${ }^{1}$ Much of this mortality and morbidity is directly influenced by the time interval between the onset of symptoms and start of treatment. Sometimes even when patients reached a referral centre, the diagnosis of ectopic pregnancy may be missed. This usually happens with subacute presentations like overdue periods, abdominal pain and abnormal uterine bleeding. Cases are often mistaken as pelvic inflammatory disease, abortions, abnormal uterine bleeding or labeled as surgical case like appendicitis. In these situations the clinical presentation is less dramatic compared to patients with acute rupture or a tubal abortion. Diagnostic tools have improved in the last two decades as well, culdocentesis, dilatation and curettage and diagnostic laparoscopy have been replaced by non- invasive diagnostic aids such as biochemical and sonographic modalities for diagnosis. These tests give rapid results and thus have helped physicians to diagnose and manage patients earlier, before it becomes a life threatening condition.

The aim of the present study was to determine the incidence of ectopic pregnancy in our hospital population and to assess the possible risk factors, clinical features and mortality rates associated with ectopic pregnancy. An attempt has been made to highlight the diagnostic errors and means to minimise them.

* Triubhuvan University Teaching Hospital, Maharajgunj, Kathmandu, Nepal.

Address for correspondence : Dr. Rajshree Jha

Dept. of Obstetrics and Gynaecology

Tribhuvan University Teaching Hospital

Maharajgunj, Kathmandu, Nepal

Email: rajshree86@hotmail.com 


\section{MATERIAL AND METHODS}

The total number of live births between April 1993 and December, 1996 was 9,063 in the department of Obstetrics and Gynaecology at Tribhuvan University Teaching Hospital. Eighty nine cases of ectopic pregnancy were recorded from the admission record in female surgical ward and the operation theatre register during this period, however only case records of 78 patients with ectopic pregnancy could be analyzed since 11 case records were missing. All these patients underwent laparotomy and the operative diagnosis of ectopic pregnancy was confirmed by histopathological evaluation of the specimen. Real time ultrasound was the most valuable diagnostic aid. The ultrasound findings of ectopic pregnancy included absence of an intra uterine gestational sac, presence of an adenexal mass and the presence of fluid in the cul-de-sac.

\section{RESULTS}

The incidence of ectopic pregnancy in this study was one $8.6 \%$ / 1000 deliveries $(0.86 \%)$ The patients ages ranged from 16- 45 with a mean of 23.9 , and 32 per cent of cases occurred in patients aged 20-24 years. [Table I] Nulliparous women constituted $29 \%$ of the cases, while women who had one or two children had the highest incidence of ectopic pregnancy in our series (45\%). Grand multi para had the lowest incidence of ectopic pregnancy $(2 \%)$, however in $6 \%$ of the cases the parity was not mentioned.

Diagnostic errors on admission occurred in $23 \%$ of the cases [table III]. Acute pelvic inflammatory disease was the commonest misdiagnosis on admission, others were labeled as tubo-ovarian mass, threatened abortion, incomplete abortion 